Patrick Oßwald, Patrick Hemberger, Thomas Bierkandt, Erdal Akyildiz, Markus Köhler, Andras Boedi, Gerber Thomas and Tina Kasper, In situ flame chemistry tracing by imaging photoelectron photoion coincidence spectroscopy, Review of Scientific Instruments, 85, 025101 (2014).

The original publication is available at http://scitation.aip.org/content/aip/journal/rsi

(C) is with AIP

http://dx.doi.org/10.1063/1.4861175 


\title{
In situ flame chemistry tracing by imaging photoelectron photoion coincidence spectroscopy
}

\author{
P. Oßwald, ${ }^{1}$ P. Hemberger, ${ }^{2}$ T. Bierkandt, ${ }^{3}$ E. Akyildiz, ${ }^{3}$ M. Köhler, ${ }^{1}$ A. Bodi, ${ }^{2}$ T. Gerber, ${ }^{2}$ \\ and T. Kasper ${ }^{3, a)}$ \\ ${ }^{1}$ German Aerospace Center (DLR) - Institute of Combustion Technology, Stuttgart 70569, Germany \\ ${ }^{2}$ Molecular Dynamics Group, Paul Scherrer Institut, CH-5232 Villigen, Switzerland \\ ${ }^{3}$ Mass Spectrometry in Reactive Flows - Thermodynamics (IVG), University of Duisburg-Essen, \\ Duisburg, Germany
}

(Received 6 September 2013; accepted 17 December 2013; published online 4 February 2014)

\begin{abstract}
Adaptation of a low-pressure flat flame burner with a flame-sampling interface to the imaging photoelectron photoion coincidence spectrometer (iPEPICO) of the VUV beamline at the Swiss Light Source is presented. The combination of molecular-beam mass spectrometry and iPEPICO provides a new powerful analytical tool for the detailed investigation of reaction networks in flames. First results demonstrate the applicability of the new instrument to comprehensive flame diagnostics and the potentially high impact for reaction mechanism development for conventional and alternative fuels. Isomer specific identification of stable and radical flame species is demonstrated with unrivaled precision. Radical detection and identification is achieved for the initial $\mathrm{H}$-abstraction products of fuel molecules as well as for the reaction controlling $\mathrm{H}, \mathrm{O}$, and $\mathrm{OH}$ radicals. Furthermore, quantitative evaluation of changing species concentrations during the combustion process and the applicability of respective results for kinetic model validation are demonstrated. Utilization of mass-selected threshold photoelectron spectra is shown to ensure precise signal assignment and highly reliable spatial profiles. (C) 2014 AIP Publishing LLC. [http://dx.doi.org/10.1063/1.4861175]
\end{abstract}

\section{INTRODUCTION}

At present, combustion accounts for $80 \%$ of the global energy consumption and will remain the dominant method of energy conversion in coming decades. ${ }^{1}$ The knowledge of how a fuel burns under various combustion conditions is a key aspect for the improvement of combustion devices with regard to future needs, e.g., varying loads, flexible fuel types, emission reductions, or efficiency increases. One prerequisite in achieving this goal is the experimental investigation of the chemical reaction network in well-defined, simple model flames and the development of chemical kinetic reaction mechanisms that describe the oxidation of the fuel and pollutant formation under these conditions. Reaction mechanisms can be validated by comparing the results of direct numerical simulations of model flames and experimentally determined species concentrations.

Flame-sampling molecular-beam mass spectrometry in combination with ionization by tunable VUV radiation from a synchrotron source (photoionization molecular-beam mass spectrometry, PI-MBMS) has proven to be an exceptionally powerful tool for the investigation of the chemical processes in laminar premixed flat flames. ${ }^{1,2}$

The reason for the success of mass spectrometric gas analysis in combustion is that mass spectrometry is one of the few techniques that is fast, sensitive, and selective enough for the analysis of flame gases without prior knowledge about the gas composition.

a) Author to whom correspondence should be addressed. Electronic mail: tina.kasper@uni-due.de
Soft, single-photon ionization with tunable VUV radiation minimizes fragmentation of flame species, facilitating the quantitative analysis of the gas composition. In addition, photoionization efficiency (PIE) curves can be measured by tuning the VUV radiation over the ionization threshold of the molecules. The thresholds and curve shapes can be compared to calibration data and provide a means of identifying individual compounds with isomeric-resolution in the complex gas mixture, specifically. .,3-5 $^{1,3}$

Currently, two nearly identical PI-MBMS instruments are in operation, one at the Advanced Light Source (ALS) in Berkeley, ${ }^{6}$ USA and the second at the National Synchrotron Radiation Laboratory (NSRL) in Hefei, ${ }^{7}$ China. Data from both instruments have been used to investigate a wide spectrum of flame chemistry issues ranging from the fuel destruction processes of hydrocarbon fuels ${ }^{2}$ and oxygenated (bio)fuels ${ }^{8}$ to the detailed analysis of the chemical processes responsible for the formation of benzene and small polyaromatic hydrocarbons (PAHs). ${ }^{1,2}$ The ability of these two stateof-the-art instruments to resolve isomer-specific concentrations and the integration of the expertise of several research groups in highly cooperative projects have led to improvements of experimental procedures and data reduction strategies that can be considered as the current benchmark for any new development in flame-sampling MBMS.

The kinetic information extractable from the comparison of experimental data in low-pressure flat flames, which are most often investigated by MBMS and numerical simulations, depends on the combined uncertainties of experiment and model. ${ }^{9-12}$ The latter include kinetic parameters, especially rate constant uncertainties, and non-kinetic uncertainties 
associated with thermodynamic and transport properties. ${ }^{13}$ The experimental uncertainties include measurements errors in gas flow rate, temperature, and species concentration. The better the qualitative and quantitative speciation data, the more suited they are to evaluate the kinetics. Consequently, PI-MBMS measurements are constantly required to improve species identification and quantification. Accuracy of species identification in PI-MBMS measurements is limited by the accuracy with which the ionization energies of isomers can be determined. Isomers can only be disentangled if their ionization thresholds differ by more than the energy resolution of the ionizing photons and their PIE curves show distinctive non-overlapping features at good signal-to-noise levels for a reliable identification of all isomers. The requirements become limiting if more than 2-3 isomers are present at a certain mass-to-charge ratio. The quantitative data reduction in PI-MBMS experiments employs a subtraction procedure to evaluate the concentrations of all isomers. For example, the quantification of fulvene and benzene requires the measurement of the fulvene signal at photon energies below the threshold of benzene and the convoluted signal at higher photon energy. The benzene signal is then obtained by subtraction of the fulvene signal weighted with the ionization cross section ratio for both photon energies. The procedure results in an accumulation of the uncertainties of the photoionization cross sections and the noise of all individual measurements.

The new instrument described in this work addresses these issues by coupling a low-pressure flat flame burner and a flame-sampling inlet to an imaging photoelectron photoion coincidence spectrometer (iPEPICO) at the Swiss Light Source. The iPEPICO detection scheme allows the measurement of photoionization mass spectra and massselected threshold photoelectron spectra (ms-TPES) in coincidence. The ms-TPES adds another analytical dimension to the species identification and can be used to measure nonconvoluted concentration profiles of isomers. The potential of the new combination of analytical techniques to the investigation of flame chemistry is discussed in this paper. Special emphasis is placed on the ability of determining branching ratios in the fuel destruction pathways, and the quantification of major and intermediate species using the photoionization mass spectra and the TPES.

\section{EXPERIMENTAL SETUP}

The setup consists of several individual components which will be described below: the low-pressure burner and burner chamber, the molecular-beam interface, and the iPEPICO spectrometer of the VUV beamline at the Paul Scherrer Institute in Villigen, Switzerland. The iPEPICO spectrometer is a permanently installed instrument and the challenge consisted in interfacing a specially designed burner chamber to the existing instrument. First measurements with the setup were conducted in May 2013 and are reported here. The complete setup is shown in Fig. 1 and a schematic drawing can be found in Fig. 2.

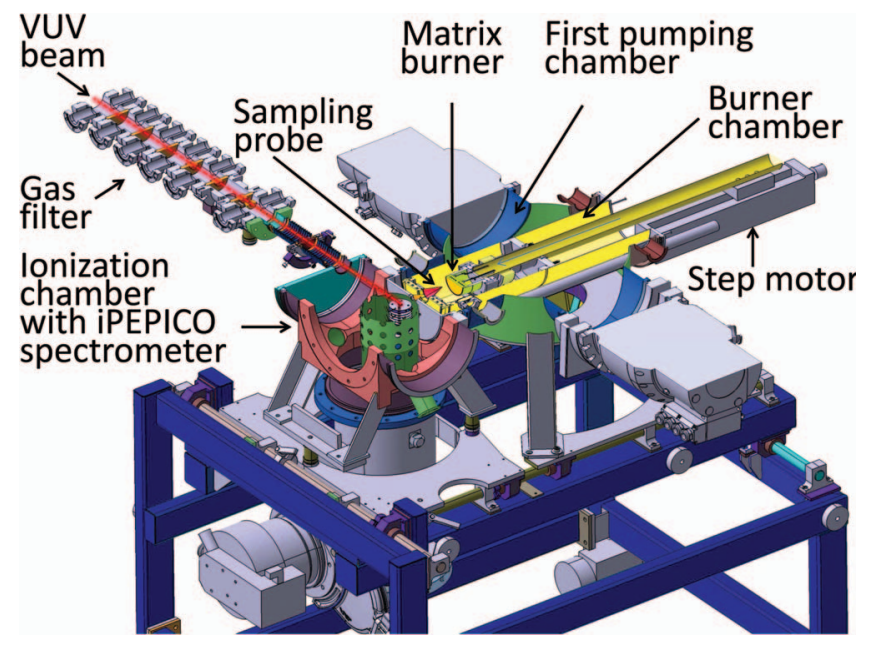

FIG. 1. Technical drawing of the complete flame-sampling instrument showing the interface between the new burner chamber and the existing iPEPICO setup and highlighting the short distance between gas extraction site and position of photoionization.

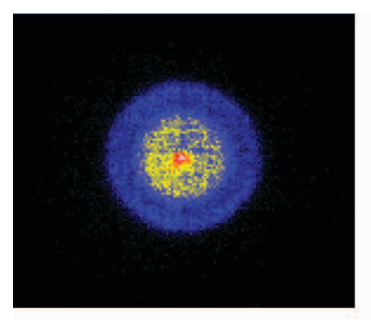

Velocity map image
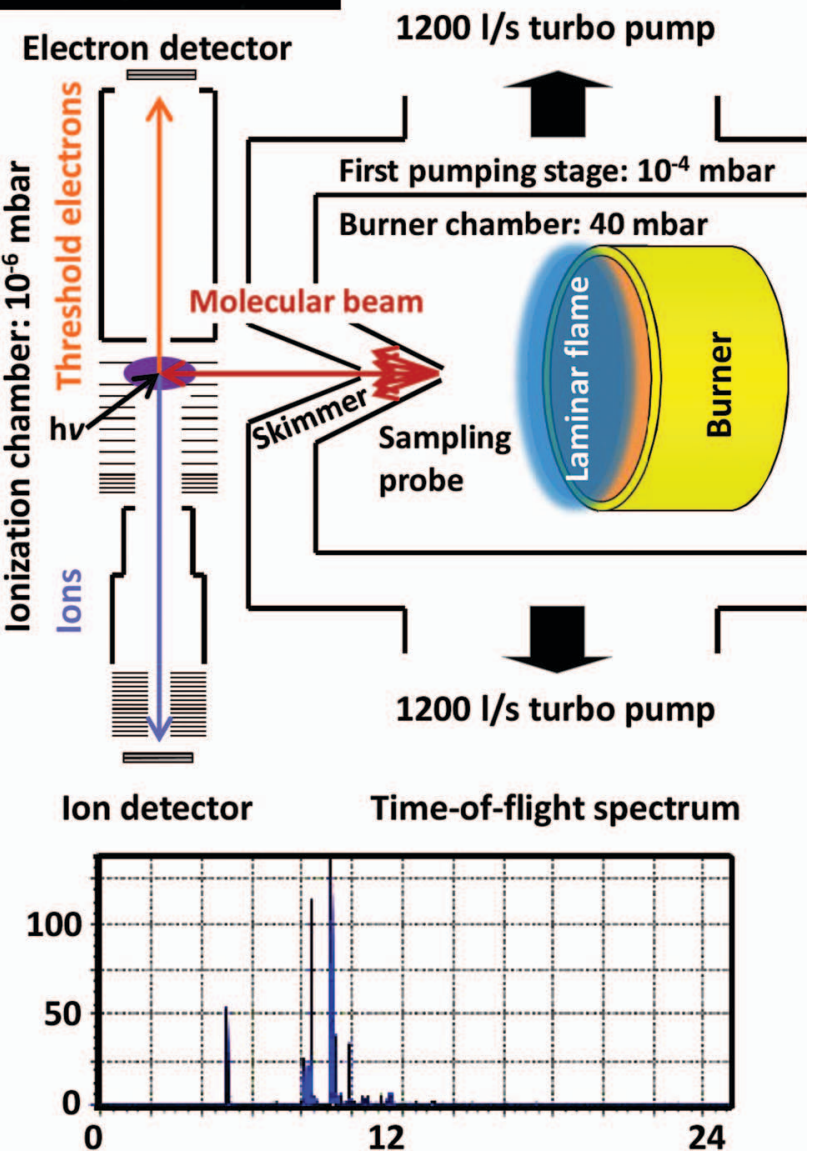

FIG. 2. Sketch of the molecular beam formation and detection schemes employed in the iPEPICO experiment at the SLS. 


\section{A. Low-pressure flame and molecular-beam sampling}

Premixed flames are stabilized on a home-built flat flame burner of McKenna type. The chamber for premixing of oxidizer and fuel is made from stainless steel and sealed by a $6.0 \mathrm{~cm}$ diameter porous bronze sinter plate. The burner is housed in a stainless steel burner chamber with a diameter of $150 \mathrm{~mm}$ and a length of $280 \mathrm{~mm}$. A conical quartz probe with a $0.5 \mathrm{~mm}$ orifice and a length of $40 \mathrm{~mm}$ is mounted to the cooled endplate of the burner chamber. The flame impinges on the end plate. Exhaust gases are pumped through four outlets spaced by $90^{\circ}$ around the burner chamber and situated close to the end plate. This setup ensures that the exhaust gases are effectively pumped from behind the sampling probe tip. Two windows at approximately the same distance from the end plate provide visual control of flame stability and probe-flame interaction. Given the constraints imposed by the existing iPEPICO spectrometer, the burner chamber had to be aligned horizontally, even though non-negligible buoyancy effects have been shown to affect measurements at large distances $(>100 \mathrm{~mm})$ between burner and sampling probe in a similar setup used for nanoparticle synthesis. ${ }^{14}$ The effect of buoyancy on measurements close to the burner is small and does not typically change flame chemistry conclusions.

The burner can be moved with respect to the sampling probe tip by a stepper motor (Nanotec ST6018L3008) driven translation stage (isel). The positioning accuracy is estimated to be $0.1 \mathrm{~mm}$.

Gas flows are metered by calibrated mass flow controllers (MKS 1179B) and the pressure in the chamber is automatically regulated by a butterfly valve (MKS 253B). The chamber is pumped by a $100 \mathrm{~m}^{3} / \mathrm{h}$ roughing pump (Oerlikon-Leybold, Sogevac 100). Several fuels and flame conditions were used in the proof-of-principle experiments and are quoted in the following paragraphs. Flames are ignited at low pressure (approximately 100 mbar) by a heating coil.

The molecular beam is extracted through the quartz probe into an intermediate pumping stage, kept at an operating pressure of typically $10^{-3}-10^{-4}$ mbar by two $1200 \mathrm{l} / \mathrm{s}$ turbo molecular pumps (Pfeiffer THP 1201, Pfeiffer TPU 1600). The core of the molecular beam passes through a nickel skimmer with $2 \mathrm{~mm}$ orifice diameter (Beam Dynamics, Inc., Model 1) into the ionization chamber of the iPEPICO spectrometer. The distance between the tip of the quartz probe and the skimmer is $72 \mathrm{~mm}$. As shown in Fig. 1, the burner chamber is inserted horizontally into the intermediate chamber. The design is a compromise that takes two conflicting requirements of flame-sampling into account. On the one hand, a rapid reduction in pressure is required to form the molecular beam and to ensure sufficient quenching of the flame reactions, so that radical species are preserved. On the other hand, the density of the molecular beam drops as $1 / r^{2}$ with increasing distance $r$ from the quartz probe. Here, we chose to maximize signal intensity at the cost of less efficient pumping of the small gap between quartz probe and skimmer and implemented a very short distance between the tip of the quartz probe and the ionization volume $(140 \mathrm{~mm})$.

The ionization chamber of the iPEPICO spectrometer is pumped by a $500 \mathrm{l} / \mathrm{s}$ turbo molecular pump (Pfeiffer, TMH
521 ) and a 1500 1/s cryo-pump (Leybold, 1500CL) to an operating pressure of $10^{-6} \mathrm{mbar}$. Quartz probe and skimmer are aligned with the ionization volume by ensuring that a laser beam passes unobstructed through all apertures and between the optics of the ion source.

\section{B. Light source and photoelectron photoion coincidence spectrometer}

The experiments were carried out at the X04DB (VUV) beamline at the Paul Scherrer Institute in Villigen, Switzerland. Since the beamline and the spectrometer were already described elsewhere, only a brief summary is given here. ${ }^{15-17}$ A water-cooled toroidal mirror collimates vacuumultraviolet light generated by a bending magnet in the storage ring. The light is diffracted by plane optical gratings, which are located in a monochromator chamber. The latter contains a turntable with three mounted gratings (150, 600 , and $1200 \mathrm{l} / \mathrm{mm}$ ). Another platinum coated toroidal mirror focuses the VUV light downstream onto the vertical slits $(200 \mu \mathrm{m})$ in the gas filter, which suppresses higher order radiation. For the 7-14 eV range, a gas mixture of neon, argon, and krypton is utilized, whereas pure neon is used between 10.5 and $21 \mathrm{eV}$. Below $7 \mathrm{eV}$ higher harmonics can be cut off by an insertable $\mathrm{MgF}_{2}$ plate. The endstation housing the iPEPICO spectrometer is directly connected to the last differentially pumped stage of the gas filter. A Wiley-McLaren ${ }^{18}$ time-of-flight (TOF) mass spectrometer $(120 \mathrm{~V} / \mathrm{cm} 1$ st extraction field, $550 \mathrm{~mm}$ drift region) is utilized to detect the ions generated in photoionization events. Electrons are velocity map imaged ${ }^{19}$ onto a Roentdek DLD40 delay line anode, which serves as a trigger for the TOF of the ion in a multiple start multiple stop approach. ${ }^{20}$ A sketch of the spectrometer is depicted in Fig. 2. For count rates up to a few tens of $\mathrm{kHz}$, each electron can be assigned to a single ionization event, enabling the measurement of ms-TPES. Threshold electrons are selected with a resolution of 5-9 $\mathrm{meV}$ and the hot electron background can be subtracted according to a literature approach. ${ }^{21}$ The photon energy was calibrated by scanning the Rydberg series of argon 11s'-13s' in both the first and second order. A resolution of around $15 \mathrm{meV}$ could be achieved with a $150 \mathrm{l} / \mathrm{mm}$ grating as measured at $15.764 \mathrm{eV}$.

Averaging times depend mainly on the concentration and the ionization cross sections of the species of interest. To obtain spectra with relevant signal-to-noise ratios $(S / N>10)$ the acquisition time for main species (mole fractions $>0.05$ ) is $30 \mathrm{~s}$ per data point, and 120-300 s for intermediates with mole fractions between $10^{-2}$ and $10^{-4}$. For species with lower mole fractions or very small ionization cross sections, typically longer averaging times are required. For example, the data of the $\mathrm{C}_{4} \mathrm{H}_{9}$ radicals in Fig. 4 were averaged for $10 \mathrm{~min}$ at each photon energy. Acquisition times exceeding $30 \mathrm{~min}$ were deemed impractical because of beamtime availability. In general, acquisitions times are comparable to other synchrotron based PI-MBMS experiments.

\section{SPECIES IDENTIFICATION}

Speciation of the multi-component gas mixture in a flame is a challenging task. Common MBMS experiments are 
able to resolve $40-60$ species $^{5,22}$ by mass separation. Isomers have the same exact mass but different chemical structures. Consequently, isomers follow different reaction pathways in flames and the resolution of their concentration profiles is crucial for the fundamental understanding of the combustion process. Mass-resolved photoionization efficiency curves obtained in PI-MBMS experiments can be used to determine ionization thresholds, but they provide only limited spectral information about the structure of the species. ${ }^{1,6,7}$ The iPEPICO/ms-TPES technique provides detailed massresolved spectral information which allows unambiguous species identifications.

\section{A. Threshold photoelectron spectra}

Measuring ms-TPE spectra has some additional advantages beyond the established isomer specific detection strategies. Vibrational transitions from the neutral to specific ionic states can be observed as progressions of resonances according to the Franck-Condon principle that reflect unique statespecific energies of an isomer yielding a fingerprint of the molecule. ${ }^{23,24}$ Such transitions can often only vaguely be observed when recording the photoion yield only. Adiabatic and vertical ionization thresholds provide another set of observables for the identification of isomers in the gas phase, which are more difficult to extract from PIE curves. Therefore, even complex isomeric compositions of reactive intermediates can be disentangled applying coincidence techniques.

Species identification by ms-TPES is demonstrated for the $\mathrm{C}_{3} \mathrm{H}_{4}$ isomers. Allene (propadiene) and propyne (methyl acetylene) are one of the smallest isomeric pairs and both species are present in most hydrocarbon flames, at least for rich conditions. Figure 3 shows the TPES measured in coincidence with $\mathrm{m} / \mathrm{z}=40$ ions in the reaction zone of a fuel-rich ethylene flame. The adiabatic ionization thresholds of both species (allene: $9.69 \mathrm{eV}$ and propyne: $10.36 \mathrm{eV}^{25}$ ) as well as the vertical values (allene: $10.02 \mathrm{eV}$ and propyne: $10.36 \mathrm{eV}^{25}$ ) are precisely determined in the flame measurements and can be used to confirm the identity of these isomers.

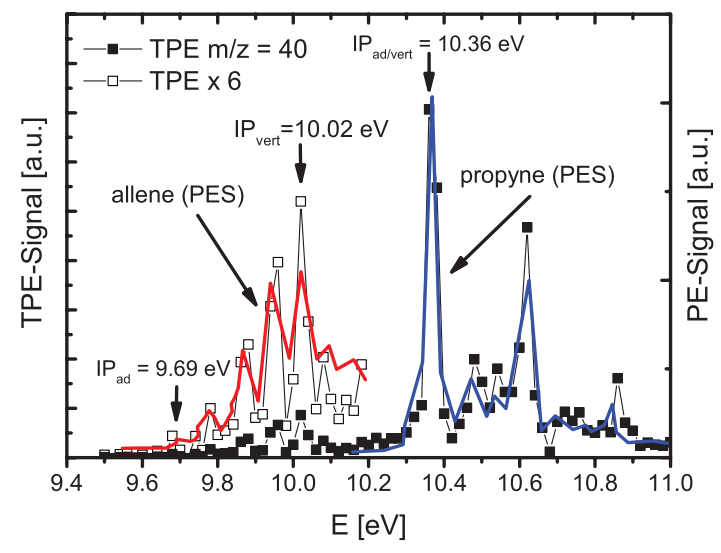

FIG. 3. Threshold photoelectron spectrum measured in coincidence with $\mathrm{m} / \mathrm{z}$ $=40$ ions (ms-TPES) sampled from the reaction zone $(h=2.8 \mathrm{~mm})$ of a fuelrich ethylene flame. Signals (symbols) are compared to photoelectron (PE) spectra (thick lines) of allene ${ }^{26}$ and propyne. ${ }^{26}$
The spectral structure of the ms-TPES in our measurements compares well to literature references obtained by photoelectron spectroscopy (PES). The $\mathrm{PES}^{26}$ of allene and propyne shown in Fig. 3 exhibit good agreement with the TPES obtained from the flame measurements. Species identity can be confirmed with a high level of confidence by the comparison of PES and ms-TPES. It should be mentioned that PE spectra are available for a large number of stable and radical species, whereas photoionization efficiency (PIE) curves, as obtained in classical PI-MBMS experiments, are rather scarce.

In typical PES experiments a high photon energy (typically obtained from the HeI resonance at $21.2 \mathrm{eV}^{26}$ ) is applied for ionization and spectra are obtained by determination of the kinetic energy distribution of the released electrons. The PES spectra in Fig. 3 have a resolution of $20-30 \mathrm{meV}$. The TPES experiment, in contrast, scans the wavelength of the ionizing light in order to detect electrons without excess energy. In the ms-TPES the resolution is determined by the resolution of the monochromator which is $15 \mathrm{meV}$ in this experiment. Both the TPES and the PES are typically in good agreement and observed peaks appear at the same energy. The obtained peak intensities may vary due to autoionization processes. However, in most cases, these effects are not important as only the energy information is used in subsequent evaluations. ${ }^{27}$ The temperature of the gas sample also has an influence on the resolution of transitions in the spectra. Photoionization is a ground-state sensitive technique and population in excited (vibrational) states can change the appearance of the spectra, e.g., due to hot-bands and sequence bands. Unfortunately, the vibrational temperatures of the literature PES are often not given (e.g., in Ref. 26) and they are unknown for the flamesampled data in this work. Rotational temperatures obtained for other low-pressure flame-sampling setups are found to be between 240 and $440 \mathrm{~K}^{28}$

While in principle it may be possible to determine the vibrational temperatures from the ms-TPES spectra, it has to be noted that molecules can be formed with vibrational excitation in flame reactions leading to population distributions that do not follow Boltzmann statistics. Determination of cooling of the internal degrees of freedom in the molecular beam when starting from unknown temperatures requires careful consideration of all parameters involved. For this reason, no attempt has been made to determine vibrational temperatures from the data in these initial experiments, precluding any discussion of features which could be assigned to hot-bands.

\section{B. Isomer-specific radical detection}

While for stable species other flame-sampling and analysis techniques (e.g., microprobe- $\mathrm{GC}^{29,30}$ ) are applicable, molecular-beam sampling is the only sampling technique that gives access to reactive species, i.e., radicals. Since combustion is driven by radical chain reactions, detailed information on the radical pool is mandatory for an understanding of combustion chemistry. Radicals present in small concentrations can lead to pollutant formation and initiate the fuel destruction in flames. The initial $\mathrm{H}$ abstraction 


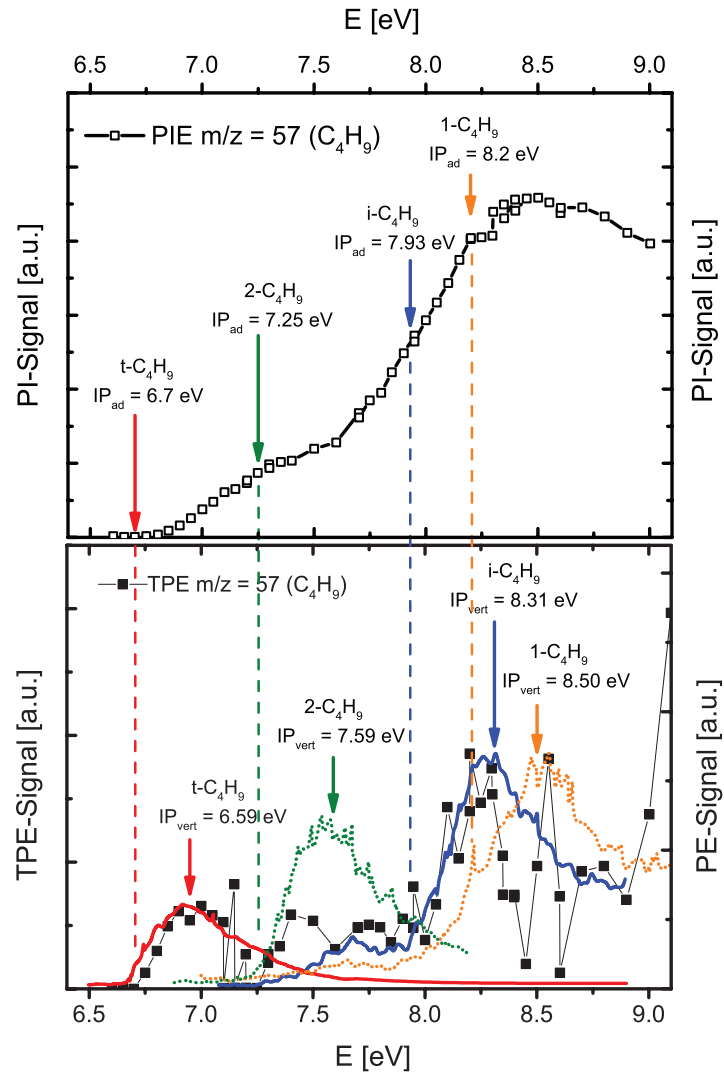

FIG. 4. PIE curve (top panel) and TPES (bottom) of the $\mathrm{m} / \mathrm{z}=57$ signal (butyl radicals) obtained from the reaction zone of a fuel-rich isobutane flame. Signals are compared to the adiabatic (top panel and dashed lines) and vertical (bottom) ionization thresholds (IP) ${ }^{25}$ and PES of tert-buty ${ }^{31}$ (solid, red line), iso-butyl ${ }^{32}$ (dotted green line), 1 -butyl ${ }^{32}$ (solid, blue line), and 2-butyl ${ }^{32}$ (dotted yellow line).

reactions from the fuel molecule leading to fuel radicals are of particular importance. Unfortunately, detection of the fuel radicals in a flame environment is extremely difficult due to their high reactivity, and the resulting low concentrations, as well as sampling losses. Furthermore, dissociative ionization of the fuel molecule can prohibit radical detection because fragments contribute to the signal at the mass of the radicals of interest. The sensitivity of the presented iPEPICO experiment, however, is high enough to overcome these challenges which can largely be attributed to the very short sample transfer distance of $140 \mathrm{~mm}$.

Signals of the fuel radicals $(\mathrm{m} / \mathrm{z}=57)$ obtained from the reaction zone of a fuel-rich isobutane flame $(\phi=1.6)$ are summarized in Fig. 4. The upper panel gives the photoionization efficiency (PIE) curve, i.e., the total ion signal recorded at $\mathrm{m} / \mathrm{z}$ $=57$ of the four butyl radical isomers as function of the photon energy. The obtained PIE scan exhibits an extraordinarily good $S / N$ ratio, especially if the low photon energies are considered. Previous investigations on a similar isobutane flame ${ }^{10}$ studied by the PI-MBMS system at the ALS were unable to obtain a PIE scan of the butyl radicals because clearly identifiable features were missing in the PIE curves. In contrast, the ms-TPES spectra show vertical transitions as peaks. If the change in the geometry from neutral to ion is large it is easier to observe the vertical transitions than the transition between the ground states as in the case of the butyl-radicals. For mod- erate changes in geometry, the transition leading to the adiabatic ionization threshold can also be observed as seen in Fig. 3 for propyne. PIE curves observe the adiabatic ionization threshold as well as vertical transitions as changes in the slope of the ion signal. As for PES or the ms-TPES the size and clarity of the step depends on the geometry change during ionization.

If reference ionization cross sections or PIE curves are not available, the identification of species relies on the comparison of individual ionization thresholds with intensity steps in the PIE curve obtained from the flame. ${ }^{3,4}$ While the approach is quite reliable for the lowest threshold in a multi compound mixture (tert-butyl radical in this case), the assignment of higher thresholds can become difficult because changes in the slopes of the PIE curves do not necessarily correlate with ionization thresholds of other isomers ${ }^{4}$ but can also be features of the PIE curves of single compounds. Direct determination of individual ionization thresholds as peaks in the ms-TPES spectra removes the ambiguity reliably.

The ms-TPES associated with the butyl radicals is shown in the lower panel of Fig. 4. Comparison with PES spectra ${ }^{31,32}$ is carried out for all possible $\mathrm{C}_{4} \mathrm{H}_{9}$ radicals: the linear isomers 1- and 2-butyl as well as the branched iso- and tert-butyl radicals. The presence of both branched isomers is clearly obvious from this analysis. The small peaks at $7.3 \mathrm{eV}$ and $8.6 \mathrm{eV}$, which could be assigned to $2-$ and $1-\mathrm{C}_{4} \mathrm{H}_{9}$, do not agree with the literature PE spectra ${ }^{32}$ and thus the presence of these isomers is unlikely. This finding is in excellent agreement with the major fuel destruction pathways for isobutane combustion ${ }^{10}$ as shown in (R1):<smiles>[CH2]C(C)C</smiles>

Beside the initial fuel radicals, the small $\mathrm{O}, \mathrm{H}$, and $\mathrm{OH}$ radicals are of crucial importance because they control reactivity. Signal contributions due to fragmentation (primarily from the high concentration product species $\mathrm{H}_{2}$ and $\mathrm{H}_{2} \mathrm{O}$ ) are a major challenge in mass spectrometric detection of these radicals, at least when electron ionization is applied. Photoionization experiments typically struggle with low signal intensities, which are caused by sampling losses and probably to a lesser degree by small absolute photoionization cross sections. For example, the cross section of the $\mathrm{H}$-atom is smaller than $6.5 \mathrm{MB}$ between threshold and $20 \mathrm{eV} .{ }^{33}$ Figure 5 shows the TPES and the PIE curves obtained for $\mathrm{m} / \mathrm{z}=1$, 16 , and 17 in a stoichiometric acetylene flame. Inferred ionization thresholds are in good agreement with literature values and spectra with excellent $S / N$ are achieved. For the $\mathrm{O}$ and $\mathrm{H}$ atoms, the line transitions can be observed at the first ionization threshold. The OH-TPES and OH-PIE curves are in excellent agreement with published PES $^{34}$ and ionization cross section $^{33}$ data. The $\mathrm{OH}$ spectra exhibit three features: 


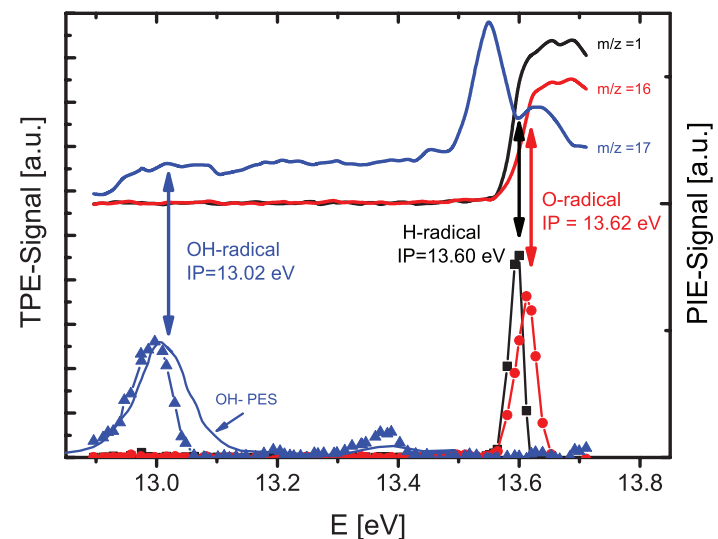

FIG. 5. TPES (symbols) and the corresponding PIE curves (thick lines) of small reactive radicals obtained from a stoichiometric acetylene flame. The ionization thresholds (IP) ${ }^{25}$ and PES of $\mathrm{OH}^{34}$ are shown, respectively.

the adiabatic ionization threshold resulting from the transition $\mathrm{OH}^{+}\left(\mathrm{X}^{3} \Sigma^{-}, v^{+}=0\right) \leftarrow \mathrm{OH}\left(\mathrm{X}^{2} \Pi, v=0\right)$ at $13.01 \mathrm{eV}$, a small peak at $13.39 \mathrm{eV}$ in the ms-TPES and a large peak in the PIE curve at $13.56 \mathrm{eV}$ which is missing in the ms-TPES. The peak at $13.39 \mathrm{eV}$ is tentatively assigned to the $\mathrm{OH}^{+}\left(\mathrm{X}^{3} \Sigma^{-}\right.$, $\left.v^{+}=1\right) \leftarrow \mathrm{OH}\left(\mathrm{X}^{2} \Pi, v=0\right)$ transition. ${ }^{35}$ The intense signal in the PIE curve at $13.56 \mathrm{eV}$ can be attributed to autoionization via $\mathrm{OH}^{*}\left(\mathrm{a}^{1} \Delta, 3 \mathrm{~d}\right)$ Rydberg states. ${ }^{36}$ Because kinetic electrons are formed during the autoionization process no signal appears in the ms-TPES spectra.

In summary, the presented iPEPICO experiment is a powerful tool for the identification of combustion intermediates. The sensitivity of the overall setup including flame-sampling MBMS system, iPEPICO spectrometer and VUV beamline is outstanding. Mass-resolved TPES, especially of radical species, can be detected with high signal-to-noise ratios and reliable identification can be achieved. These spectra have a high potential to enable significant progress in understanding molecular fuel destruction reactions.

\section{QUANTITATIVE SPECIES PROFILES}

For an increased impact on combustion mechanism development, quantitative data must be obtained from the msTPES. In order to trace the progress of chemical reactions occurring in the combustion process, spatially resolved measurements are conducted. So-called "burner-scans" are produced by incrementally increasing the distance between burner and sampling cone at a distinct photon energy. For proper interpretation of the results or for validation of kinetic reaction models, spatial profiles must provide quantitative information, i.e., mole fractions. These can be derived from the signal ratios of individual ms-TPE peaks if proper cross section or calibration data are available. The following section demonstrates a quantification approach for the presented iPEPICO experiment.

\section{A. Major species and kinetic modeling}

Quantification of the major combustion species, i.e., reactants, products, and dilutants follow an internal calibration strategy widely used for fuel-rich low-pressure flames in various MBMS experiments. ${ }^{5,6,11,22,37}$ The same strategy is applicable in the present study, even though signal proportionalities for the iPEPICO experiment differ slightly from other MBMS systems (see Sec. IV B for details).

For fuel-rich conditions, the product species are $\mathrm{H}_{2}, \mathrm{H}_{2} \mathrm{O}$, $\mathrm{CO}, \mathrm{CO}_{2}$, and Ar. Their exhaust gas mole fractions (distance above the burner, $h=30 \mathrm{~mm}$ ) are typically determined using an internal calibration strategy based on the elemental $\mathrm{C}, \mathrm{H}$, and $\mathrm{O}$ balances and the determination of the $\mathrm{CO} / \mathrm{CO}_{2}$ ratio. A similar approach is used to determine the composition at the burner surface (i.e., the first measurement position; $h \approx 0 \mathrm{~mm}$ ) applying the $\mathrm{O}$ and $\mathrm{C}$ balance. To account for early consumption of small amounts of reactants near the burner surface, contributions from $\mathrm{CO}, \mathrm{CO}_{2}$, and $\mathrm{H}_{2} \mathrm{O}$ are considered. A detailed description of the evaluation procedure is provided as supplementary material. ${ }^{62}$

In order to demonstrate that quantification is possible for iPEPICO flame data, we have chosen a fuel-rich ethylene/oxygen/Ar flame (18.75/31.25/50.0 mol.\%). The pressure in the flame chamber was set to $40 \mathrm{mbar}$ and the total flow rate was $4 \mathrm{slm}$ (standard liter per minute). Major species concentrations were derived from burner scans with second harmonic radiation at $18 \mathrm{eV}$. Note that none of the major species is ionized at $9 \mathrm{eV}$. The $\mathrm{CO} / \mathrm{CO}_{2}$ exhaust gas ratio was calculated with literature ionization cross sections at $18 \mathrm{eV}^{38,39}$ Due to the mass overlap of ethylene with $\mathrm{CO}$, the ethylene profile is derived from the $\mathrm{m} / \mathrm{z}=27$ fragment signal using the partial ionization cross sections for the $\mathrm{C}_{2} \mathrm{H}_{4}{ }^{+}$and $\mathrm{C}_{2} \mathrm{H}_{3}{ }^{+}$ ionization channels of ethylene. ${ }^{40}$ No further corrections (e.g., background subtraction) were necessary. The resulting mole fraction profiles are presented in Fig. 6.

The experimental results are compared to a onedimensional flame simulation using a detailed reaction mechanism from Miller et al. ${ }^{41}$ The mechanism contains 123 species and 783 reversible and partially pressure dependent reactions including enol chemistry. It was validated amongst others fuels for ethylene flames and low-pressure conditions.

Calculations were performed using the premixed burner stabilized flame module of the DARS software. ${ }^{42}$ Transport properties were treated as mixture average diffusion, including thermal diffusion. An experimental temperature profile

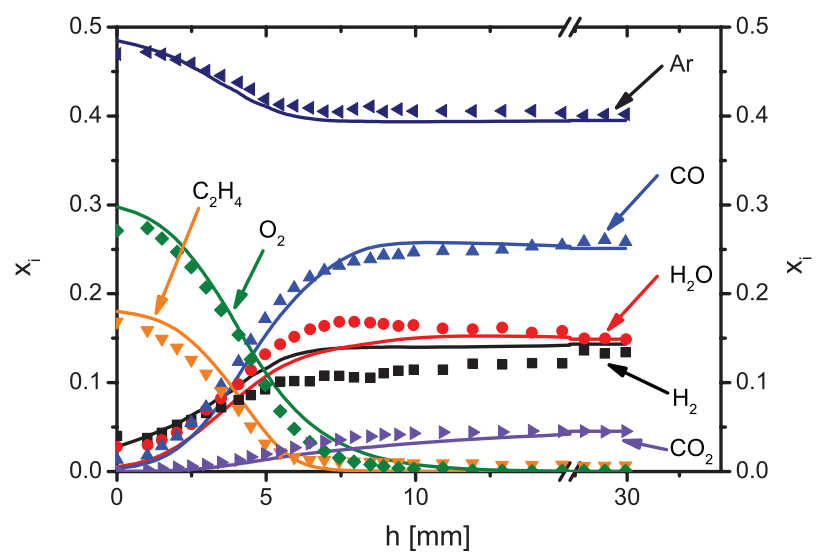

FIG. 6. Spatially resolved mole faction profiles of the major species measured (symbols) in a fuel-rich ethylene flame and comparison with the result of a chemical kinetic reaction model ${ }^{41}$ (lines). 
was used as input parameter instead of solving the energy equation. The temperature profile was obtained from the sampling rate following procedures described previously ${ }^{11,43}$ based on the pressure in the 1st pumping stage. The resulting "disturbed" temperature profile partially accounts for influences of the sampling probe and has been shown to be a useful approximation for modeling MBMS data. ${ }^{9-12,44-46}$ The profiles determined with the above procedure depend on the exhaust gas temperature for calibration. For the flame conditions in this experiment it has not been measured yet and was estimated to be $2200 \mathrm{~K}$ based on similar flames. ${ }^{11,41}$ The modeling results obtained for the major species are in excellent agreement with the measurements as shown in Fig. 6.

Profile shapes match perfectly for $\mathrm{C}_{2} \mathrm{H}_{4}, \mathrm{O}_{2}, \mathrm{CO}, \mathrm{CO}_{2}$, and $\mathrm{Ar}$. The experimental data reduction and modeling was done independently and no optimization was performed to improve the agreement, e.g., by variation of the estimated temperature. Only the $\mathrm{H}_{2}$ - and $\mathrm{H}_{2} \mathrm{O}$-profiles deviate slightly in the post flame zone. However, the agreement obtained for this dataset is comparable to results obtained by other MBMS experiments, e.g., Refs. 44-50.

Data reduction strategies typically rely on instrumentspecific and time-consuming calibration measurements that coevolve with the needs of the flame chemistry measurements. Given the initial character of these proof-of-principle experiments the remarkable agreement between model and experiment is encouraging and uncertainties can be further reduced, for example, by the more exact determination of the exhaust gas $\mathrm{CO} / \mathrm{CO}_{2}$ ratio and the experimental determination of the exhaust gas temperature.

\section{B. Intermediate species}

In contrast to the major species calculation, quantification strategies for intermediates must be modified to reflect the characteristics of the iPEPICO experiment. The dependency of the total ion signal $S_{i}$ on experimental parameters in the present experiment may be written as

$$
S_{i}=x_{i}(h) \cdot D_{i}\left(M_{i}\right) \cdot t \cdot F K T(T, \bar{M}) \cdot c(E) \cdot \sigma_{i}(E) \cdot \alpha_{i}(E-I P) .
$$

With $x_{i}$ being the mole fraction of the species $i$ at a specific flame position $h ; D$ is a mass $M$ dependent discrimination factor, accounting for potential loss of light species in the molecular beam; $t$ is total time of signal accumulation; FKT is a composition- and thus position-dependent sampling function; $c$ is a device-specific constant combining various experimental constants, for the present experiment a finite energy $E$ dependence of $c$ has to be considered due to grating efficiency and stability; $\sigma_{\mathrm{i}}$ is the partial ionization cross section, i.e., the cross section for a specific $\mathrm{m} / \mathrm{z}$ signal; $\alpha$ is a correction factor for signal loss due to limited energy range of electron imaging detector.

In comparison to evaluation approaches for other MBMS experiments, ${ }^{4,11}$ some modifications must be introduced to account for the continuous ionization and the electron/ion coincidence arrangement. A major limitation is the finite size of the electron detector. Electrons with high kinetic energy can have a high enough velocity orthogonal to the detector axis porting them beyond the sensitive area. This process leads to a partial loss of ion signal related to those fast electrons. In our setup, this loss becomes effective for measurements performed with a high gap $(>1.2 \mathrm{eV})$ between the adiabatic ionization threshold ( $\mathrm{IP}_{\mathrm{ad}}$ ) and the actual photon energy. We have accounted for this effect by introduction of a species and energy dependent factor $\alpha$, with $0<\alpha \leq 1$. Close to the ionization threshold all photoelectrons are captured and $\alpha$ becomes unity.

Quantification of any intermediate can be achieved if the parameters listed in Eq. (1) are known. They can be obtained following approaches developed for quantification of other PI-MBMS data. ${ }^{4,9,51}$ A detailed description of the procedure is provided in the supplementary material. ${ }^{62}$ However, tabulated cross sections are not applicable in the case of incomplete electron detection, i.e., the parameter $\alpha(\mathrm{E})$ cannot be determined easily. Consequently, all species are evaluated near their ionization thresholds (i.e., E-IP $<1.2 \mathrm{eV}$ ). A stable photon flux also has to be maintained because variations in flux were not recorded in these initial experiments. Burner scans are obtained for photon energies of 10.75, 10.57, 10.22, and $9.52 \mathrm{eV}$. The absolute uncertainty of the obtained mole fractions is estimated to be at the same level than for PI-MBMS data, i.e., below a factor 2 depending on the quality of the cross section data used in the analysis.

Figure 7 summarizes the experimental results for the $\mathrm{C}_{3} \mathrm{H}_{4}$ isomers and 1,3-butadiene obtained from the ethylene flame, and the model predictions for those species. The 1,3butadiene mole fraction was obtained from the $9.52 \mathrm{eV}$ burner scan with the ionization cross section from Ref. 52. As for the major species, the quantitative agreement of model and experiment is excellent apart from a small spatial shift between the profiles. Previously, results ${ }^{41}$ obtained with the same model and using undisturbed temperature profiles needed an arbitrary shift to obtain a correct spatial dependence. The smallest photon energy used in this study was $9.52 \mathrm{eV}$. Consequently, the $\mathrm{C}_{4} \mathrm{H}_{6}$ mole fraction value reflects the accumulated uncertainty of the progressive quantification from high energy scans to low energy scans (see the supplementary material). ${ }^{62}$

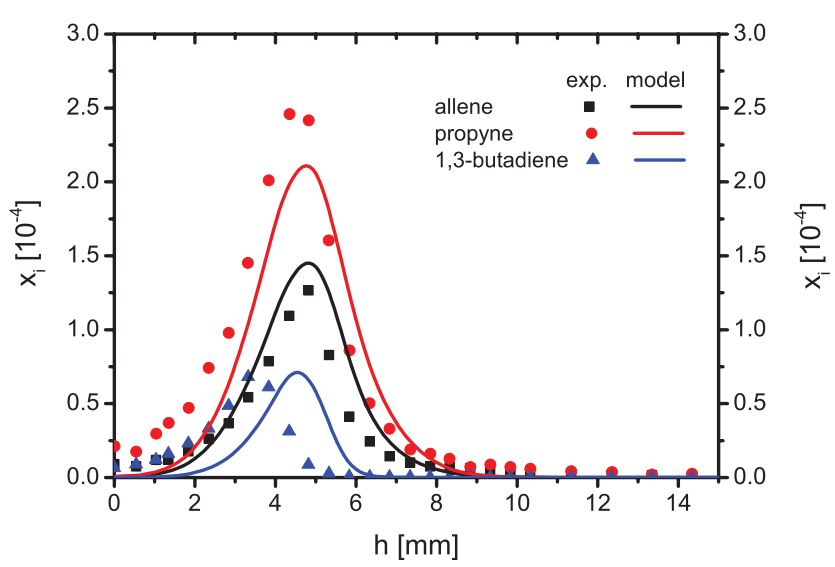

FIG. 7. Spatial mole fraction profiles of the $\mathrm{C}_{3} \mathrm{H}_{4}$ isomers and butadiene measured (symbols) in a fuel-rich ethylene flame and comparison with the model $^{41}$ result (lines). 
The good agreement between experiment and simulation confirms the validity of the evaluation approach for the iPEPICO flame data.

In addition, the quantitative profiles for both $\mathrm{C}_{3} \mathrm{H}_{4}$ isomers are depicted in Fig. 7. Allene (propadiene) was obtained from the $10.22 \mathrm{eV}$ scan using the ionization cross section in Ref. 53. Since this photon energy is below the ionization threshold of propyne (methyl acetylene, IP $=10.36 \mathrm{eV}^{25}$ ), the signal at $10.22 \mathrm{eV}$ is exclusively due to allene. The propyne mole fraction is evaluated from the $10.57 \mathrm{eV}$ burner scan. At this energy, both isomers contribute to the ion signal and the signal fraction of allene was subtracted prior to the quantitative evaluation of propyne. The signal contribution of allene was obtained calculating backwards from the known mole fraction and its ionization cross section at $10.57 \mathrm{eV}^{53}$

Again, almost ideal agreement with the model prediction is obtained for both isomers validating the chosen evaluation approach. Additionally, the direct transferability of procedures originating from PI-MBMS experiments, ${ }^{4,51}$ to the total ion signal obtained in the iPEPICO setup is confirmed. The peak mole fractions of most other species evaluated in this ethylene flame do not deviate more than a factor of $2-3$ from the respective model predictions; this is commonly considered as fair agreement. The results demonstrate the general capabilities of the iPEPICO technique for model validation purposes. Quantitative species profiles can be provided with at least comparable quality to other photoionization experiments when the total ion signal is considered. However, monitoring of species profiles measured in coincidence with threshold photoelectrons raises an additional analytic dimension. Specific opportunities of these TPE profiles are discussed in Sec. IV C.

\section{Quantification of threshold electron coincidence ion signals}

The quantitative isomer separation based on total ion signals as described above and applied in PI-MBMS experiments employs a subtraction procedure. As demonstrated, the quantification of allene and propyne requires the measurement of the allene signal at photon energies below the threshold of propyne and the convoluted signal at higher photon energy. The propyne signal is then obtained by subtraction of the allene signal weighted with the ionization cross section ratio for both photon energies. The procedure results in the accumulation of the uncertainties of the (three) photoionization cross sections and of the noise of all individual measurements.

Data quality can be improved if the quantitative species profiles are determined from ion signal measured in coincidence with threshold electrons, i.e., electrons without kinetic energy. For burner scans recorded at the exact photon energy of a specific molecular transition, i.e., at the peak wavelength of the respective TPES, the coincidence ion signal is dominated by the species of interest. Profiles can be measured with a high level of confidence since potential signal contributions from other species with the same $m / z$ ratio or fragment ions can be identified in the TPES and are negligible as long as the TPES are structured interpretably. In particular, no signal corrections must be applied if isomers with lower ion-
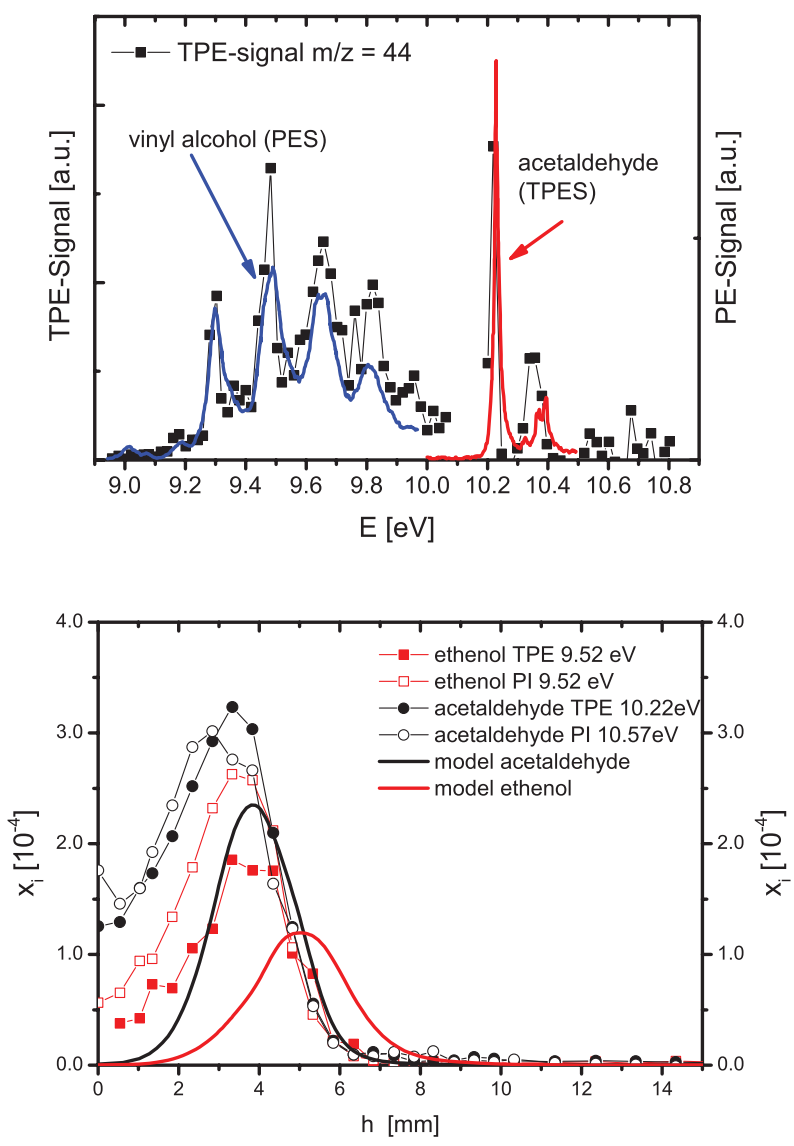

FIG. 8. TPES (top panel) and mole fraction profiles (bottom panel) of the $\mathrm{C}_{2} \mathrm{H}_{4} \mathrm{O}$ isomers obtained from the total ion signal (PI) and from threshold electron signals (TPE). TPE signals are compared to the literature PE spectrum of vinyl alcohol ${ }^{60}$ and TPE spectrum of acetaldehyde. ${ }^{61}$

ization threshold are present in the flame. Consequently, no additional uncertainties related to the use of literature cross sections are introduced. In the following, the $\mathrm{C}_{2} \mathrm{H}_{4} \mathrm{O}$ isomers are used to demonstrate the advantages.

Both $\mathrm{C}_{2} \mathrm{H}_{4} \mathrm{O}$ isomers, namely acetaldehyde and its tautomer vinyl alcohol (ethenol), have been detected in many flames. Vinyl alcohol and other enols were discovered to play a role in oxidation chemistry of flames in $2005^{54}$ when synchrotron-based photoionization MBMS was implemented as diagnostic tool. Enol chemistry is currently being incorporated into some kinetic reaction models. ${ }^{9}{ }^{41}$ The lower panel of Fig. 8 shows the TPES of $m / z=44$ obtained in the reaction zone $(h=2.8 \mathrm{~mm})$ of the ethylene flame discussed above. Individual scans are measured for both isomers and the spectrum of vinyl alcohol is abitrarily scaled in the figure to facilitate comparison. The presence of both isomers is obvious from comparison of the flame-sampled and literature spectra. Resonances in the TPES, i.e., at $9.52 \mathrm{eV}$ for vinyl alcohol and at $10.22 \mathrm{eV}$ for acetaldehyde, are chosen as photon energies in the burner scans.

Quantification of the raw data profiles can be achieved following the same strategy as for the total ion signal. However, a state-selective ionization cross section has to be used in Eq. (1) to quantify the TPE signal. For vinyl alcohol the appropriate cross section is accessible by scaling the absolute cross $\operatorname{section}^{53}$ with the ratio of threshold/total-ion 
signal, derived from the $m / z=44$ TPES flame data. Because both species contribute to the total ion signal at $10.22 \mathrm{eV}$, a similar simple calculation is not possible for acetaldehyde. In a first approximation, the partial ionization cross section for the transition at $9.22 \mathrm{eV}$ was assumed to correlate with the inflection point of the first step in the total ionization cross section $(2.5 \mathrm{Mb}) .{ }^{53}$ Since the selected energy corresponds to the first transition in the acetaldehyde TPES and the recorded peak shape is almost symmetric, this approximation may be plausible. However, this strategy will not be transferable to other situations and cold gas reference measurements will be needed for reliable quantification.

The mole fraction profiles obtained from the TPE signals are compared to the results obtained from the total ion signals and the corresponding modeling result in the lower panel of Fig. 8. The observed agreement of both profiles is satisfactory. The deviation between the peak mole fractions obtained for vinyl alcohol is less than $30 \%$, which is a typical deviation when different quantification strategies are compared. The fact that these measurements were performed very close to the ionization threshold of vinyl alcohol, where the ionization cross section is small and small signal contributions from other sources (fragmentation, isotope contribution, insufficient higher harmonic elimination, etc.) would strongly influence the PI signal, may serve as explanation for the slightly higher mole fraction. The good agreement of the acetaldehyde mole fractions is less significant than it appears because rough estimations have been made for the TPE quantification. However, the peak shape is in perfect agreement.

The agreement with the model prediction is also acceptable even if the model slightly underestimates both $\mathrm{C}_{2} \mathrm{H}_{4} \mathrm{O}$ isomer mole fractions. The concentration ratio of both isomers is predicted correctly, with better agreement for the TPE results. The slight deviation in the profile position is similar to the observation made for the $\mathrm{C}_{3} \mathrm{H}_{4}$ isomers.

In conclusion, species profiles obtained from the TPE signal can be quantified with similar or even less uncertainty than signals from the total ion signal when proper reference spectra are available. Since the TPE signal is restricted to a specific molecular transition, signal interference, for example, due to fragmentation, is minimal if the TPES is structured.

\section{FLAME AS RADICAL SOURCE}

The burner setup developed for our experiments could be used as a highly effective source of elusive radicals, which can be subject to spectroscopic studies to determine their ionization energies and vibrational levels. A flame apparatus can complement pyrolysis, discharge, and photolysis sources, since combustion leads to a wide spectrum of intermediates such as radicals and carbenes without the need for specific radical precursors. The extraordinary selectivity of massselected TPES eliminates the need of a clean, well characterized environment for a fundamental spectroscopic assessment. However, the poor definition of the internal energy of flame-sampled species may be a drawback of burner sources.

Under fuel-rich conditions the propargyl radical, a benzene precursor molecule, is abundant in many flames with mole fractions in the order of $10^{-3}-10^{-4}$. Figure 9 shows a

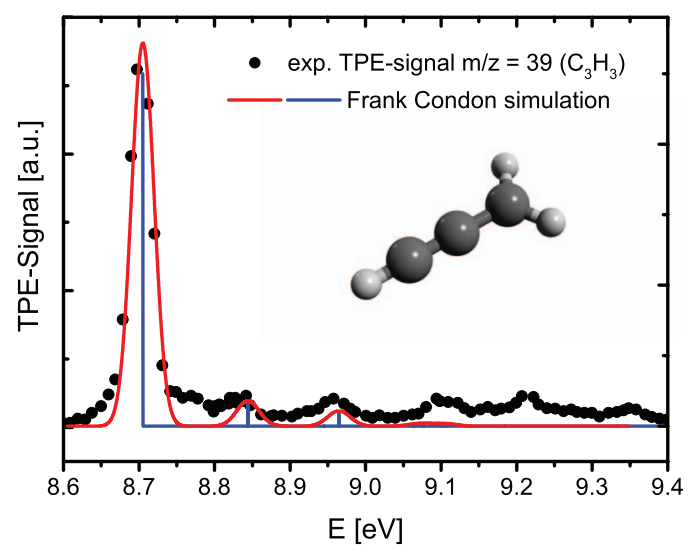

FIG. 9. Mass-selected threshold photoelectron spectrum of the propargyl radical measured in an isobutane flame compared to a Franck-Condon simulation from Ref. 55.

typical ms-TPES of the propargyl radical as measured in an isobutane flame of stoichiometry $\phi=1.6$ recorded at $h=$ $4.3 \mathrm{~mm}$ above the burner surface. Position and intensities of the bands at $8.70,8.84$, and $8.86 \mathrm{eV}$ match perfectly with the Franck-Condon simulation carried out by Botschwina et al. ${ }^{55}$ and other experimental data. ${ }^{56,57}$ These bands correspond to the adiabatic ionization energy $\left(\mathrm{IP}_{\mathrm{ad}}\right)$ and transitions into the pseudosymmetric $\left(v_{5}\right)$ and antipseudosymmetric $\left(v_{3}\right) \mathrm{CC}$ stretching vibrations of the ion. The features at 9.1 and $9.2 \mathrm{eV}$ correspond to higher vibrationally excited states of the ion and are underestimated in the simulation. These transitions become more intense in a TPE spectrum because autoionizing Rydberg states ${ }^{58}$ lie in the same energy region of the rovibrational ion states. ${ }^{59}$

The total time to measure such a spectrum amounts to a few hours, showing the large abundance of free radicals in a burner and the high efficiency of the sampling interface and spectrometer.

\section{CONCLUSIONS}

We report the first successful coupling of a flat flame burner with a flame-sampling molecular-beam interface to an imaging photoelectron photoion coincidence spectrometer at the VUV beamline at the Swiss Light Source. The iPEPICO detection scheme allows the simultaneous determination of photoionization mass spectra and TPES adding another analytical dimension to the investigation of chemical combustion processes. The potential of the new combination of analytical techniques to advance the investigation of flame chemistry is demonstrated for ethylene, acetylene, and isobutane flames.

The main benefits of the new analytical technique include species identification with superior precision in the determination of ionization thresholds, even in the presence of lower ionizing species. The setup allows for the detection and precise identification of stable and reactive species (including fuel radicals) sampled from the reaction zone of a flat flame. Superior sensitivity, high signal-to-noise ratio, and detection without interference of dissociative ionization channels are shown by high quality $\mathrm{O}, \mathrm{H}$, and $\mathrm{OH}$ spectra. Special emphasis is placed on the potential of determining 
branching ratios in the fuel destruction pathways. Exemplary isomer separation was demonstrated for the different butyl radicals produced in an isobutane flame.

In addition to the superior identification capabilities of the iPEPICO technique, quantitative determination of spatially resolved species profiles was demonstrated. Quantification analysis strategies for this new experiment pose new challenges that are addressed and major and intermediate species mole fraction profiles of an ethylene flame are reported.

Furthermore, the general capabilities of the iPEPICO experiment for model validation purposes are highlighted. The obtained species profiles are reproduced by a kinetic reaction model with comparable quality as known from common MBMS experiments. Beyond the quantification of the photoionization mass spectra, evaluation of ms-TPE signals is found to provide superior isomer resolving qualities due to the omission of deconvolution procedures.

In summary, the presented combination of flamesampling and coincidence spectroscopy is shown to be a powerful tool for the investigation of fundamental combustion reaction networks. Application of the setup as radical source for spectroscopic studies of molecular properties is suggested.

\section{ACKNOWLEDGMENTS}

The iPEPICO experiments were carried out at the VUV beamline of the Swiss Light Source, Paul Scherrer Institute. The authors are grateful for CAD support by S. Sonderegger and L. Han. Equipment left to P.O. and T.K. by P. Bisling has been used and was greatly appreciated. Able technical support by the machine shop of the University of DuisburgEssen was essential to the experiment. T.B., E.A., and T.K. are grateful for financial support from the MIWF of the state of North Rhine-Westphalia. Funding by the Helmholtz Association is acknowledged by P.O. and M.K. P.H., A.B., and T.G. acknowledge funding by the Swiss Federal Office for Energy (BFE Contract No. 101969/152433).

${ }^{1}$ F. Qi, Proc. Combust. Inst. 34, 33 (2013).

${ }^{2}$ N. Hansen, T. A. Cool, P. R. Westmoreland, and K. Kohse-Höinghaus, Prog. Energy Combust. Sci. 35, 168 (2009).

${ }^{3}$ A. Lucassen, P. Oßwald, U. Struckmeier, K. Kohse-Höinghaus, T. Kasper, N. Hansen, T. A. Cool, and P. R. Westmoreland, Proc. Combust. Inst. 32, 1269 (2009).

${ }^{4}$ T. Kasper, P. Oßwald, U. Struckmeier, K. Kohse-Höinghaus, C. A. Taatjes, J. Wang, T. A. Cool, M. E. Law, A. Morel, and P. R. Westmoreland, Combust. Flame 156, 1181 (2009).

${ }^{5}$ P. Oßwald, H. Güldenberg, K. Kohse-Höinghaus, B. Yang, T. Yuan, and F. Qi, Combust. Flame 158, 2 (2011).

${ }^{6}$ T. A. Cool, A. McIlroy, F. Qi, P. R. Westmoreland, L. Poisson, D. S. Peterka, and M. Ahmed, Rev. Sci. Instrum. 76, 094102 (2005).

${ }^{7}$ F. Qi, R. Yang, B. Yang, C. Huang, L. Wei, J. Wang, L. Sheng, and Y. Zhang, Rev. Sci. Instrum. 77, 084101 (2006).

${ }^{8}$ K. Kohse-Höinghaus, P. Oßwald, T. A. Cool, T. Kasper, N. Hansen, F. Qi, C. K. Westbrook, and P. R. Westmoreland, Angew. Chem., Int. Ed. 49, 3572 (2010).

${ }^{9}$ S. M. Sarathy, S. Vranckx, K. Yasunaga, M. Mehl, P. Oßwald, W. K. Metcalfe, C. K. Westbrook, W. J. Pitz, K. Kohse-Höinghaus, R. X. Fernandes, and H. J. Curran, Combust. Flame 159, 2028 (2012).

${ }^{10} \mathrm{P}$. Oßwald, K. Kohse-Höinghaus, U. Struckmeier, T. Zeuch, L. Seidel, L. Leon, and F. Mauss, Z. Phys. Chem. 225, 1029 (2011).

${ }^{11}$ M. Schenk, L. Leon, K. Moshammer, P. Oßwald, T. Zeuch, L. Seidel, F. Mauss, and K. Kohse-Höinghaus, Combust. Flame 160, 487 (2013).
${ }^{12}$ A. Frassoldati, R. Grana, T. Faravelli, E. Ranzi, P. Oßwald, and K. KohseHöinghaus, Combust. Flame 159, 2295 (2012).

${ }^{13}$ G. P. Smith, in Applied Combustion Diagnostics, edited by K. KohseHöinghaus and J. B. Jeffries (Taylor \& Francis, 2002).

${ }^{14}$ C. Weise, A. Faccinetto, S. Kluge, T. Kasper, H. Wiggers, C. Schulz, I. Wlokas, and A. Kempf, Combust. Theory Modell. 17, 504 (2013).

${ }^{15}$ A. Bodi, P. Hemberger, T. Gerber, and B. Sztaray, Rev. Sci. Instrum. 83, 083105 (2012).

${ }^{16}$ A. Bodi, M. Johnson, T. Gerber, Z. Gengeliczki, B. Sztaray, and T. Baer, Rev. Sci. Instrum. 80, 034101 (2009).

${ }^{17}$ M. Johnson, A. Bodi, L. Schulz, and T. Gerber, Nucl. Instrum. Methods Phys. Res. A 610, 597 (2009).

${ }^{18}$ W. C. Wiley and I. H. McLaren, Rev. Sci. Instrum. 26, 1150 (1955).

${ }^{19}$ B. J. Whitaker, Imaging in Molecular Dynamics (Cambridge University Press, Cambridge, 2003).

${ }^{20}$ A. Bodi, B. Sztaray, T. Baer, M. Johnson, and T. Gerber, Rev. Sci. Instrum. 78, 084102 (2007).

${ }^{21}$ B. Sztàray and T. Baer, Rev. Sci. Instrum. 74, 3763 (2003).

${ }^{22}$ T. Kasper, U. Struckmeier, P. Oßwald, and K. Kohse-Höinghaus, Proc. Combust. Inst. 32, 1285 (2009).

${ }^{23}$ A. Bodi, P. Hemberger, D. L. Osborn, and B. Sztáray, J. Phys. Chem. Lett. 4, 2948 (2013).

${ }^{24}$ P. Hemberger, A. J. Trevitt, E. Ross, and G. da Silva, J. Phys. Chem. Lett. 4, 2546 (2013).

${ }^{25}$ S. G. Lias, J. E. Bartmess, J. F. Liebman, J. L. Holmes, R. D. Levin, S. A. Kafafi, and W. G. Mallard, NIST Chemistry WebBook, NIST Standard Reference Database Number 69 (National Institute of Standards and Technology, Gaithersburg, MD, 2013), see http://webbook.nist.gov.

${ }^{26}$ K. Kimura, S. Katsumata, Y. Achiba, T. Yamazaki, and S. Iwata, Handbook of HeI Photoelectron Spectra of Fundamental Organic Molecules (Japan Scientific Societies Press, Tokyo, 1981).

${ }^{27}$ J. Harvey, P. Hemberger, A. Bodi, and R. P. Tuckett, J. Chem. Phys. 138, 124301 (2013).

${ }^{28}$ M. Kamphus, N. N. Liu, B. Atakan, F. Qi, and A. McIlroy, Proc. Combust. Inst. 29, 2627 (2002).

${ }^{29}$ R. Hertz, T. Streibel, C. Liu, K. McAdam, and R. Zimmermann, Anal. Chim. Acta 714, 104 (2012).

${ }^{30}$ E. Pousse, P. A. Glaude, R. Fournet, and F. Battin-Leclerc, Combust. Flame 156, 954 (2009).

${ }^{31}$ F. A. Houle and J. L. Beauchamp, J. Am. Chem. Soc. 101, 4067 (1979).

${ }^{32}$ J. C. Schultz, F. A. Houle, and J. L. Beauchamp, J. Am. Chem. Soc. 106, 3917 (1984)

${ }^{33}$ H. P. Palenius, J. L. Kohl, and W. H. Parkinson, Phys. Rev. A 13, 1805 (1976).

${ }^{34}$ S. Katsumata and D. R. Lloyd, Chem. Phys. Lett. 45, 519 (1977).

${ }^{35}$ J. D. Barr, A. De Fanis, J. M. Dyke, S. D. Gamblin, N. Hooper, A. Morris, S. Stranges, J. B. West, and T. G. Wright, J. Chem. Phys. 110, 345 (1999).

${ }^{36}$ F. Innocenti, L. Zuin, M. L. Costa, A. A. Dias, A. Morris, A. C. S. Paiva, S. Stranges, J. B. West, and J. M. Dyke, J. Electron Spectrosc. Relat. Phenom. 142, 241 (2005).

${ }^{37}$ Y. Li, L. Zhang, Z. Tian, T. Yuan, J. Wang, B. Yang, and F. Qi, Energy Fuels 23, 1473 (2009)

${ }^{38}$ G. R. Wight, M. J. Van Der Wiel, and C. E. Brion, J. Phys. B 9, 675 (1976).

${ }^{39}$ A. P. Hitchcock, C. E. Brion, and M. J. van der Wiel, Chem. Phys. 45, 461 (1980).

${ }^{40}$ R. A. Mackie, S. W. J. Scully, A. M. Sands, R. Browning, K. F. Dunn, and C. J. Latimer, Int. J. Mass Spectrom. 223-224, 67 (2003).

${ }^{41}$ C. A. Taatjes, N. Hansen, J. A. Miller, T. A. Cool, J. Wang, P. R. Westmoreland, M. E. Law, T. Kasper, and K. Kohse-Höinghaus, J. Phys. Chem. A 110, 3254 (2006).

${ }^{42}$ DARS, Lund Combustion Engineering, Dars Digital Analysis of Reaction Systems.

${ }^{43}$ U. Struckmeier, P. Oßwald, T. Kasper, L. Böhling, M. Heusing, M. Köhler, A. Brockhinke, and K. Kohse-Höinghaus, Z. Phys. Chem. 223, 503 (2009).

${ }^{44}$ D. Liu, C. Togbé, L. S. Tran, D. Felsmann, P. Oßwald, P. Nau, J. Koppmann, A. Lackner, P. A. Glaude, B. Sirjean, R. Fournet, F. Battin-Leclerc, and K. Kohse-Höinghaus, Combust. Flame (in press).

${ }^{45}$ L. S. Tran, C. Togbé, D. Liu, D. Felsmann, P. Oßwald, P. A. Glaude, R. Fournet, B. Sirjean, F. Battin-Leclerc, and K. Kohse-Höinghaus, Combust. Flame (in press).

${ }^{46}$ C. Togbé, L. S. Tran, D. Liu, D. Felsmann, P. Oßwald, P. A. Glaude, B. Sirjean, R. Fournet, F. Battin-Leclerc, and K. Kohse-Höinghaus, Combust. Flame (in press). 
${ }^{47}$ V. Dias, C. Duynslaegher, F. Contino, J. Vandooren, and H. Jeanmart, Combust. Flame 159, 1814 (2012).

${ }^{48}$ I. E. Gerasimov, D. A. Knyazkov, S. A. Yakimov, T. A. Bolshova, A. G Shmakov, and O. P. Korobeinichev, Combust. Explos. Shock Waves 48 , 661 (2012).

${ }^{49}$ N. Hansen, S. S. Merchant, M. R. Harper, and W. H. Green, Combust. Flame 160(11), 2343-2351 (2013).

${ }^{50}$ Z. Wang, Y. Li, F. Zhang, L. Zhang, W. Yuan, Y. Wang, and F. Qi, Proc. Combust. Inst. 34, 1785 (2013).

${ }^{51}$ P. Osswald, U. Struckmeier, T. Kasper, K. Kohse-Höinghaus, J. Wang, T. A. Cool, N. Hansen, and P. R. Westmoreland, J. Phys. Chem. A 111, 4093 (2007).

${ }^{52}$ T. A. Cool, J. Wang, K. Nakajima, C. A. Taatjes, and A. McIlroy, Int. J. Mass Spectrom. 247, 18 (2005).

${ }^{53}$ T. A. Cool, K. Nakajima, T. A. Mostefaoui, F. Qi, A. McIlroy, P. R. Westmoreland, M. E. Law, L. Poisson, D. S. Peterka, and M. Ahmed, J. Chem. Phys. 119, 8356 (2003).

${ }^{54}$ C. A. Taatjes, N. Hansen, A. McIlroy, J. A. Miller, J. P. Senosiain, S J. Klippenstein, F. Qi, L. Sheng, Y. Zhang, T. A. Cool, J. Wang, P. R.
Westmoreland, M. E. Law, T. Kasper, and K. Kohse-Höinghaus, Science 308, 1887 (2005).

${ }^{55}$ P. Botschwina, R. Oswald, and G. Rauhut, Phys. Chem. Chem. Phys. 13, 7921 (2011).

${ }^{56}$ P. Hemberger, M. Lang, B. Noller, I. Fischer, C. Alcaraz, B. K. Cunha de Miranda, G. A. Garcia, and H. Soldi-Lose, J. Phys. Chem. A 115, 2225 (2011).

${ }^{57}$ H. Gao, Y. Xu, L. Yang, C. S. Lam, H. Wang, J. Zhou, and C. Y. Ng, J. Chem. Phys. 135, 224304 (2011).

${ }^{58}$ J. D. Savee, S. Soorkia, O. Welz, T. M. Selby, C. A. Taatjes, and D. L. Osborn, J. Chem. Phys. 136, 134307 (2012).

${ }^{59}$ A. J. Yencha, A. J. Cormack, R. J. Donovan, A. Hopkirk, and G. C. King, Chem. Phys. 238, 109 (1998).

${ }^{60}$ G. Y. Matti, O. I. Osman, J. E. Upham, R. J. Suffolk, and H. W. Kroto, J. Electron Spectrosc. Relat. Phenom. 49, 195 (1989).

${ }^{61}$ A. J. Yencha, M. R. F. Siggel-King, G. C. King, A. E. R. Malins, and M. Eypper, J. Electron Spectrosc. Relat. Phenom. 187, 65 (2013).

${ }^{62}$ See supplementary material at http://dx.doi.org/10.1063/1.4861175 for a detailed description of quantitative data evaluation procedure. 